\title{
Qualitative Analyzing and Evaluating Energy Efficient Datacenters Based on Cloud Model in Cloud Environment
}

\author{
Ke Han ${ }^{1, \mathrm{a}}$, Hui Rong ${ }^{1, \mathrm{c}}$ \\ ${ }^{1}$ Kunming Metallurgy College, Kunming 650300, China \\ ahanke81@qq.com, ${ }^{\mathrm{C}} 573233998 @ q q . c o m$
}

\author{
Xiaobo $\mathrm{Cai}^{2 *, \mathrm{~b}}$ \\ ${ }^{2}$ Yunnan Agricultural University, Kunming 650021, China \\ bxbcai918@qq.com
}

\begin{abstract}
The green cloud becomes a center of attention, how to evaluate the energy efficiency of the cloud environment has been one of difficult and attractive field . Cloud models are the effective tools in transforming between qualitative concepts and their quantitative expressions. This paper designs an uncertainty enhanced energy-efficiency evaluation model based on cloud model theory, through the cloud model we convert the energy, performance and QoS parameters into qualitative concept, and then we propose a new tasks scheduling algorithm to optimize the energy-efficiency of cloud datacenters. Our research results can be integrated into Cloud systems to monitor energy efficiency and support static or dynamic system-level energy-efficiency optimization.
\end{abstract}

Keywords-Green cloud, energy efficiency, cloud model, tasks scheduling

\section{INTRODUCTION}

Cloud computing delivers computing as a utility to users worldwide. The Cloud technology has become the reference paradigm for the realization of large-scale storage and computational services. A consequence of this paradigm is that cloud data centers have high deployment and operational costs, as well as significant carbon footprints for the environment. We need to develop Green Cloud Computing(GCC) solutions that reduce these deployment and operational costs and thus save energy and reduce adverse environmental impacts. As one of the critical steps in the processes of energy efficiency evaluation, weight decision for energy efficiency evaluation factors, whose accuracy has direct impact on green grading, can be recognized as one of the core actions in such work. Generally, the ways currently adopted to measure weight include faxtor paired comparison method, principal component annlysis, etc., all of them are based on exactly collected energy efficiency parameters, and the final result is calculated through mathematic way. Whereas real cloud environment is a nonlinear and dynamic uncertain system, containing multiple parameters, the data used to describe the energy efficiency are also uncertain, thus leads to hard description for real cloud environment in precision with conventional mathematical model. A cloud model, as a quality-quantity interchangeable model combining traditional fuzzy mathematics and probability and statistics, realizes their conversion between qualitative words and quantitative values. Based on the cloud model and correlation analysis, taking deliberate consideration of the fuzziness and randomness existing in objects and human knowledge, In order to achieve this objective, a thorough understanding of the energy efficiency patterns in complex Cloud environments is needed. this paper success fully builds the conversion model and proposes a new way for weight decision of energy efficiency evaluation factors.

\section{RELATED WORKS}

As cloud and green computing paradigms are closely related and they are gaining their momentum, the energy efficiency of clouds has become one of most crucial research issues. There have been many studies of energy efficiency evaluating for the cloud environment and many algorithms for improving the energy efficiency. Advancements in hardware technologies [1], such as low-power CPUs, solid state drives, and energy-efficient computer monitors have helped relieve this energy issue to a certain degree. Although thermal-aware load balancing or scheduling does not seem to be directly related to energy consumption, the cooling (air-conditioning) in these computing facilities consumes a considerable amount of energy [2, 3]. In [3] and [4], the main focus is rather on temperature and/or heat emissions of resources in data centers. In the meantime, there also have been a considerable amount of research conducted using software approaches, such as scheduling and resource allocation [5-9] and task consolidation [10-13]. The scheduling and resource allocation approach is primarily enabled using slack reclamation with the support of dynamic voltage/frequency scaling (DVFS; more spherically 'processor undervaluing') [14] incorporated into many recent commodity processors. This technique temporarily decreases voltage supply level at the expense of lowering processing speed. Since most DVFS-based energy-aware scheduling and resource allocation techniques are static (off line) algorithms with an assumption of tight coupling between tasks and resources (i.e., local tasks and dedicated resources), their application to our cloud scenario is not apparent, if not possible. In the task consolidation approach, virtualization technologies play a key role. 
These technologies with the prevalence of many-core processors have greatly eased and boosted parallel processing; that is, a single many-core processor often runs multiple tasks (processes or threads) concurrently. This parallel processing practice at a glance seems to inherently increase performance and productivity. This fact has much motivated many task consolidation studies [10-13]. Task consolidation in[10]is approached using the traditional bin-packing problem with two main characteristics, i.e., CPU and disk usage. The Virtual Power approach proposed in 13] incorporates task consolidation into its power management combining 'soft' and 'hard' scaling methods. These two methods are based on power management facilities (e.g., resource usage control method and DVFS) equipped with virtual machines (VMs) and physical processors, respectively.

Firstly, we use cloud model to express the factor's evaluation standard of the appointed datacenter style according to the expert's knowledge and experience by means of the cloud model description method in Section 4

\section{ClOUd MODEL}

Cloud model is an uncertainty conversion model between some qualitative concept expressed with Linguistic words and its relative quantitative value .Given a qualitative concept $T$ defined over a universe of discourse $U$, let $X \in U$ is a random instantiation of the concept $T$ and $\mu . T(x) \in[0,1]$ is the certainty degree of $X$ belonging to $T$, which corresponds to a random number with a steady tendency. Then, the distribution of $X$ in the universe $U$ can be defined as a cloud and ${ }^{X}$ can be called as a cloud drop. A cloud describes the overall quantitative property of a concept by the three numerical characteristics as follows: Expectation $E x$ is the mathematical expectation of the cloud drops belonging to a concept in the universal. Entropy $E n$ represents the uncertainty measurement of a qualitative concept. It is determined by both the randomness and the fuzziness of the concept. In one aspect, as the measurement of randomness, $E n$ reflects the dispersing extent of the cloud drops and in the other aspect, it is also the measurement of fuzziness, representing the scope of the universe that can be accepted by the concept. Hyper entropy $H e$ is the uncertain degree of entropy $E n$.

\section{DEscription Of EVAluation Standard OF ENERGY EFFICIENCY EVALUATION FACTORS WITH CLOUd MODEL}

The energy efficiency of datacenter is mapped as green degree of some cloud environment, then there is a positive relation between energy efficiency degree and factors which influence datacenters energy efficiency. Before analyzing the relation between energy efficiency and evaluation factors, we can translate the uncertain factor conditions into quantitative impact values which energy efficiency evaluation factor impacts on green cloud with the uncertain illation based on cloud model. Take the factor "QoS level” as example, we can establish the qualitative illation rule by the knowledge of expert as follow:

If SLA "higher", then impact value "higher";

If SLA "high", then impact value "high"

If SLA “common", then impact value "common"

If SLA "low", then impact value "low"

If SLA "lower", then impact value"lower"

The description methods of the five qualitative concepts of the rule's front with cloud model in distance field are described as foregoing. Using the cloud of the rule's front to drive forward cloud generator, we can get a group of distance figures, then get their average to be the input of condition $X$ cloud generator, and count the certainty degrees of the five qualitative concepts of the rule's front respectively, we can find the maximum. For energy efficiency of data centers in cloud system, we choose energy consumption, QoS and performance to be the evaluation factors, every factor include some specific sub-factors.

TABLE 1 EVALUATION STANDARD OF ENERGY EFFICIENCY EVALUATION FACTORS

\begin{tabular}{lllllll}
\hline Factor & Sub-factor & \multicolumn{5}{c}{ Evaluation standard } \\
\hline \multirow{3}{*}{ Energy consumption } & ECPU & Higher & High & Common & Low & lower \\
& ERAM & Higher & High & Common & Low & lower \\
& EMem & Higher & High & Common & Low & lower \\
& & Higher & High & Common & Low & lower \\
QoS & SLAratio & Higher & High & Common & Low & lower \\
Performance & Processingtime & Higher & High & Common & Low & lower \\
& Utilization & Higher & High & Common & Low & lower \\
& & & & & & \\
\hline
\end{tabular}

Table 1 shows the evaluation standards of the energy efficiency for datacenters in cloud environments. In Table 1 , the evaluation standard of every standard of every evaluation factors are according with the knowledge of experts respectively. We use a qualitative and fuzzy language value to express a certain numerical range. From this special sequence, the normal cloud model can represent together by Entropy with randomness and fuzziness and form a model represented by uncertain knowledge. For a stochastic variable which strictly obey the normal distribution, if the expectation has been given, the conjugate prior distribution of the deviation is the reciprocal- $\gamma$ distribution. Design a special algorithm; create a new stochastic variable through a random deviation.

There are several cloud computing categories with this work focused on a public cloud .A public cloud is based on the standard cloud computing model, with service provides by a service provider. The cloud model does not emphasize the description of accurate function, but represents uncertainty using three digital characters. The cloud generator is the basis of doing uncertainty illation by cloud model. Connecting a condition $X$ cloud generator and a condition $Y$ cloud generator can make a single rule generator. 
algorithm 1 cloud drops generate algorithm

Input: the quantitative value of $\mathrm{N}$ cloud drops, and uncertain degrees of each cloud drop representing the concept $\tilde{A}$. Output: three digital characters, which represents a qualitative concept and the number of cloud drops.

1. create a normal stochastic variable $y=\exp \left[-\left(x-E_{X}\right)^{2} / 2\left(E_{n}^{\prime}\right)^{2}\right]$, whose expectation is $E_{n}$, and standard deviation is $H_{e}$.

2. create a normal stochastic variable $\mathrm{x}$ with its expectation is $E_{x}$, and standard deviation abs $\left(E_{n}^{\prime}\right)$.

3. make $x$ be a cloud drop as a concrete quantitative value of qualitative concept $\tilde{A}$.

4. calculate $\gamma$.

5. make $y$ as a certain degree of x belongs to the qualitative concept $\tilde{A}$.

$\{x, y\}$ reflects the whole contents of this changing.

6.To repeat (1) to (6) until $N$ cloud drops are generated.

\section{RESOURCE SCHEDULING AlgORITHM}

maximize resource utilization, ultimately to minimize

The task consolidation problem in this study is the process of assigning a set $N$ of $n$ tasks (service requests or simply services) to a set $N$ of $n$ cloud nodes aiming to

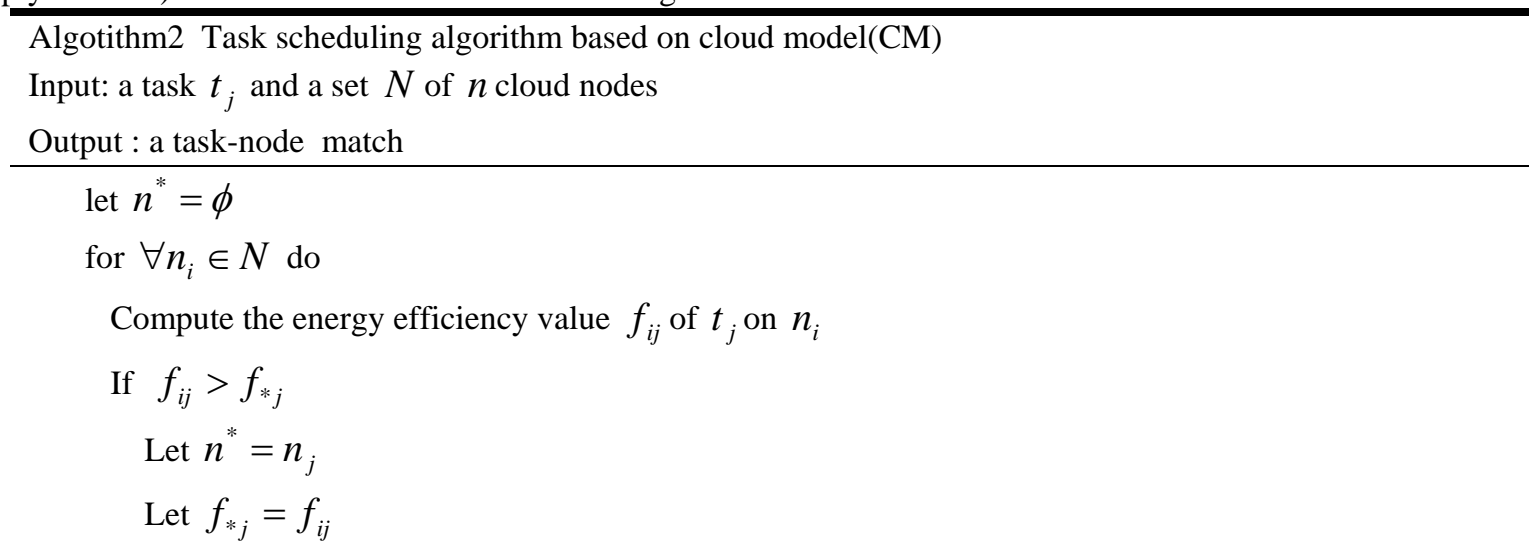

End if

End for

Assign $t_{j}$ to $n^{*}$

\section{EVALUATION}

For the benchmark policies we simulated Optimize Response Time (OPT) , Reconfigure Dynamically with Load(RDL) and the Task scheduling based on cloud model(CM) . The proposed heuristics have been evaluated by simulation using CloudSim toolkit [5]. The simulated data center comprises 10 heterogeneous physical nodes. Each node is modeled to have one CPU core with performance equivalent to 1000, 2000 or 3000 MIPS, 8 GB of RAM and 1 TB of storage. Users submit requests for provisioning of 50 heterogeneous VMs that fill the full capacity of the data center. The simulation results presented in Fig1 show that dynamic reallocation of tasks according to current energy efficiency value brings higher energy savings compared with static allocation policies.

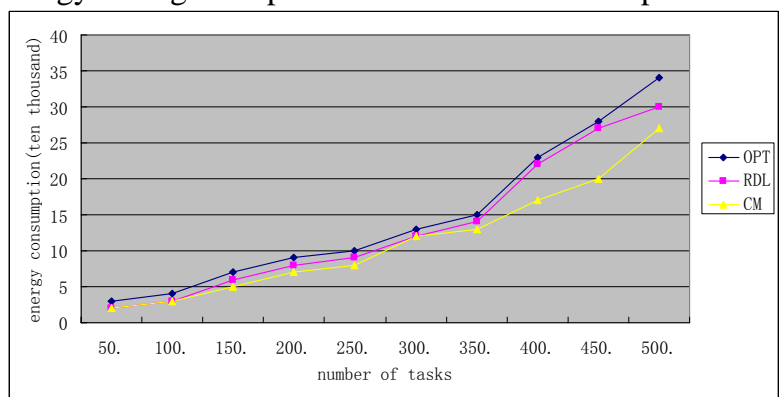

Figure 1. Simulation Results using different tasks consolidation algorithms 


\section{CONCLUSION AND FUTURE WORK}

The normal cloud model is an uncertain transforming model described by the language value that can transform between a qualitative concept and its quantitative expression. We use it to transform accurate numerical values to qualitative language values efficiently. In this work we have proposed and evaluated heuristics for dynamic reallocation of tasks to minimize energy consumption, while providing reliable QoS and performance. The obtained results show that the technique of dynamic reallocation of tasks based on energy efficiency cloud model brings substantial energy savings and is applicable to real-world Cloud data centers. For the future work, we propose to investigate the consideration of Heterogeneous cloud environment tasks in reallocation decisions to the overall energy efficiency.

\section{ACKNOWLEDGMENT}

This research was supported by with the nature and science fund from YunNan province ministry of education of China (No.: $2014 Z 076$ No.:2014Y494) .

\section{REFERENCES}

[1] Venkatachalam V, Franz M (2005) Power reduction techniques for microprocessor systems. ACM Comput Surv 37(3):195-237

[2] Koomey JG (2007) Estimating total power consumption by servers in the U.S. and the world. Lawrence Berkeley National Laboratory, Stanford University

[3] Tang Q, Gupta SK, Varsamopoulos G (2008) Energy-efficient thermal-aware task scheduling for homogeneous high-performance computing data centers: a cyber-physical approach. IEEE Trans Parallel Distrib Syst 19(11): 1458-1472

[4] Moore J, Chase J, Rangana than P, Sharma R (2005) Making scheduling cool: temperature-aware workload placement in data centers. In: Proc USENIX annual technical conference
[5] LeeYC, Zomaya AY(2009)Minimizing energy consumption for precedence-constrained applications using dynamic voltage scaling. In: Proc the international symposium on cluster computing and the grid (CCGRID ’09), 2009, pp 92-99

[6] Kim KH, Buyya R, Kim J (2007) Power aware scheduling of bag-of-tasks applications with deadline constraints on DVS-enabled clusters. In: Proc seventh IEEE international symposium on cluster computing and the grid (CCGrid '07), 2007, pp 541-548

[7] Zhu D, Melhem R, Childers BR (2003) Scheduling with dynamic voltage/speed adjustment using slack reclamation in multiprocessor real-time systems. IEEE Trans Parallel Distrib Syst 14(7):686- 700

[8] Ge R, Feng X, Cameron KW (2005) Performance-constrained distributed DVS scheduling for scientific applications on power-aware clusters. In: Proc the ACM/IEEE conference on supercomputing (SC ’05), 2005, pp 34-44

[9] Chen JJ, Kuo TW (2005) Multiprocessor energy-efficient scheduling for real-time tasks with different power characteristics. In: Proc international conference on parallel processing (ICPP '05), 2005, pp 13-20

[10] Srikantaiah S, Kansal A, Zhao F (2008) Energy aware consolidation for cloud computing. In: Proc USENIX workshop on power aware computing and systems in conjunction with OSDI, 2008, pp 1-5

[11] Song Y, Zhang Y, Sun Y, Shi W (2009) Utility analysis for internet-oriented server consolidation in VM-based data centers. In: Proc IEEE international conference on cluster computing (Cluster '09), 2009

[12] Torres J, Carrera D, Hogan K, Gavalda R, Beltran V, Poggi N (2008) Reducing wasted resources to helpachieve greendatacenters.In: Proc 4th workshop on high-performance, power-aware computing (HPPAC '08), 2008

[13] Nathuji R, Schwan K (2007) VirtualPower: coordinated power management in virtualized enterprise systems. In: Proctwenty-first ACMSIGOPS symposium on operating systems principles (SOSP'07), 2007, pp 265-278

[14] Khan SU, Ahmad I (2009) A cooperative game theoretical technique for joint optimization of energy consumption and response time in computational grids. IEEE Trans Parallel Distrib Syst 21(4):537553 